\title{
Fundamental domains and generators for lattice Veech groups
}

\author{
Ronen E. Mukamel*
}

\begin{abstract}
The moduli space $Q \mathcal{M}_{g}$ of non-zero genus $g$ quadratic differentials has a natural action of $G=\mathrm{GL}_{2}^{+}(\mathbb{R}) /\left\langle \pm\left(\begin{array}{ll}1 & 0 \\ 0 & 1\end{array}\right)\right\rangle$. The Veech group $\operatorname{PSL}(X, q)$ is the stabilizer of $(X, q) \in$ $Q \mathcal{M}_{g}$ in $G$. We describe a new algorithm for finding elements of $\operatorname{PSL}(X, q)$ which, for lattice Veech groups, can be used to compute a fundamental domain and generators. Using our algorithm, we give the first explicit examples of generators and fundamental domains for non-arithmetic Veech groups where the genus of $\mathbb{H} / \operatorname{PSL}(X, q)$ is greater than zero.
\end{abstract}

Mathematics Subject Classification (2010). 32G15, 30F30.

Keywords. Riemann surfaces, Teichmüller theory, Veech groups.

\section{Introduction}

Fix an integer $g \geq 2$ and let $\mathcal{M}_{g}$ be the moduli space of genus $g$ Riemann surfaces. The space $\mathcal{M}_{g}$ is a complex orbifold and carries a complete, Finsler Teichmüller metric whose geodesics are explicitly described by Teichmüller's theorem. The bundle $Q \mathcal{M}_{g} \rightarrow \mathcal{M}_{g}$ of holomorphic quadratic differentials consists of pairs $(X, q)$ where $q$ is a non-zero holomorphic quadratic differential on $X \in \mathcal{M}_{g}$. The geodesic flow on $\mathcal{M}_{g}$ gives an $\mathbb{R}$-action on $Q \mathcal{M}_{g}$ which, together with the $\mathbb{C}^{*}$-action fixing $X$ and rescaling $q$, generates an action of $G=\mathrm{GL}_{2}^{+}(\mathbb{R}) /\left\langle \pm\left(\begin{array}{ll}1 & 0 \\ 0 & 1\end{array}\right)\right\rangle$ on $Q \mathcal{M}_{g}$.

The Veech group $\operatorname{PSL}(X, q)$ is the stabilizer of $(X, q)$ in $G$. There are many examples of surfaces whose Veech groups are known to be complicated $[7,8,11,17]$. There are few examples of Veech groups that have been described in their entirety. For instance, each integer $D \geq 5$ congruent to 0 or $1 \bmod 4$ determines a quadratic differential $\left(X_{D}, q_{D}\right)$ in $Q \mathcal{M}_{2}$ as in Figure 3 whose Veech group is a lattice in $\mathrm{PSL}_{2}(\mathbb{R})[5,10]$. The homeomorphism type of $\mathbb{H} / \operatorname{PSL}\left(X_{D}, q_{D}\right)$ is determined in $[2,12,13]$ but little else is known about $\operatorname{PSL}\left(X_{D}, q_{D}\right)$ outside of some small values for $D$.

The purpose of this paper is to introduce girth differentials and use them to study the group $\operatorname{PSL}(X, q)$ and the quotient $\mathbb{H} / \operatorname{PSL}(X, q)$. We will describe an algorithm

\footnotetext{
${ }^{*}$ The research for this paper was supported in part by grant DMS-1103654 from the National Science Foundation.
} 
for finding elements of $\operatorname{PSL}(X, q)$ which, for lattice Veech groups, gives generators for $\operatorname{PSL}(X, q)$ and a fundamental domain for the action of $\operatorname{PSL}(X, q)$ on $\mathbb{H}$. Using an implementation of our algorithm in Sage [14], we compute the first explicit examples of fundamental domains and generators for non-arithmetic Veech groups where the genus of $\mathbb{H} / \operatorname{PSL}(X, q)$ is greater than zero.

Theorem 1.1. Fundamental domains and generators for $\operatorname{PSL}\left(X_{D}, q_{D}\right)$ are encoded in Table 1 for $D=44,45,48,52,53,56,57$, and 60.

The discriminant $D=44$ is the smallest discriminant giving rise to a positive genus quotient $\mathbb{H} / \operatorname{PSL}\left(X_{44}, q_{44}\right)$ (the genus is one). In Figure 1 we give a fundamental domain for $\operatorname{PSL}\left(X_{44}, q_{44}\right)$. It is straightforward to check whether any given element $g \in G$ is in $\operatorname{PSL}\left(X_{D}, q_{D}\right)$ by comparing the Delaunay triangulations of $\left(X_{D}, q_{D}\right)$ and $g \cdot\left(X_{D}, q_{D}\right)$. Since the Delaunay decomposition of a quadratic differential is unique, $g$ is in $\operatorname{PSL}\left(X_{D}, q_{D}\right)$ if and only if the Delaunay polygons for $\left(X_{D}, q_{D}\right)$ differ from those for $g \cdot\left(X_{D}, q_{D}\right)$ by translations respecting the gluing relations. To prove Theorem 1.1 without refering to the results in this paper, one simply checks that the claimed generators are in $\operatorname{PSL}\left(X_{D}, q_{D}\right)$ and that the group they generate has covolume equal to the volume of $\mathbb{H} / \operatorname{PSL}\left(X_{D}, q_{D}\right)$, which is computed in [2]. We will give another proof of Theorem 1.1, at the end of this introduction, that is independent of [2]. The rest of this paper is devoted to explaining the algorithm used to generate Table 1 .

Girth differentials and the well-girthed spine. Throughout this paper, we fix a quadratic differential $\left(X_{0}, q_{0}\right)$ in $Q \mathcal{M}_{g}$ and set $Q L=G \cdot\left(X_{0}, q_{0}\right)$ and $L=\mathbb{C}^{*} \backslash Q L$. The quotient $L$ is a hyperbolic Riemann surface isomorphic to $\mathbb{H} / \operatorname{PSL}\left(X_{0}, q_{0}\right)$ and points in $L$ are quadratic differentials in $Q L$ up to scale.

We will call a differential $(X, q) \in Q L$ a girth differential if one of its shortest saddle connections is horizontal of length one and for each $(X, q) \in Q L$ we define:

$$
N(X, q)=\left\{\lambda \in \mathbb{C}^{*}:(X, \lambda q) \text { is a girth differential }\right\} .
$$

The set $N(X, q)$ is a non-empty, cyclically ordered and finite set. The number of elements in $N(X, q)$ depends only on the differential up to scale $[X, q] \in L$ and equals the product of the number of girth differentials in $\mathbb{C}^{*} q$ with the orbifold order of $[X, q]$ in $L$. We will call a differential up to scale $[X, q] \in L$ well-girthed if \#N(X,q) $\geq 2$.

Our algorithm for finding elements of $\operatorname{PSL}\left(X_{0}, q_{0}\right)$ is based on the study of the level sets for $\# N(X, q)$ :

$$
L[n]=\{[X, q] \in L: \# N(X, q)=n\},
$$

and the well-girthed spine:

$$
\operatorname{Spine}_{W G}(L)=\bigcup_{n \geq 2} L[n] .
$$


Our main theorem shows that the sets $L[n]$ stratify $L$ by easy to understand pieces and $L$ deformation retracts onto $\operatorname{Spine}_{W G}(L)$.

Theorem 1.2. The sets $L[n]$ give a stratification $L=\bigcup_{n \geq 1} L[n]$ by disjoint sets with $\bigcup_{n \geq k} L[n]$ closed for each $k \geq 1$ and $L$ deformation retracts ${ }^{1}$ onto its well-girthed spine:

$$
L \rightarrow \operatorname{Spine}_{W G}(L) .
$$

Each connected component of L[1] is diffeomorphic to the disk or the punctured disk, each connected component of L[2] is a finite length geodesic arc and the set $\bigcup_{n \geq 3} L[n]$ is discrete in $L$.

In particular, the well-girthed spine $\operatorname{Spine}_{W G}(L)$ is homeomorphic to a graph. Examples well-girthed spines are depicted in Figures 1 and 4.

Finite volume orbits. When the volume of $L$ is finite, we use the Veech dichotomy to show that $L[1]$ contains a neighborhood of each cusp, allowing us to prove:

Theorem 1.3. If the volume of $L$ is finite, then $\operatorname{Spine}_{W G}(L)$ is compact, the set $\bigcup_{n \geq 3} L[n]$ is finite, the set $L[2]$ has finitely many connected components and the set $L[1]$ consists of \# cusps $(L)$ components of each of which is diffeomorphic to the punctured disk.

For finite volume $L$, the well-girthed spine is homeomorphic to a finite graph.

Decorated Riemann surfaces. Theorem 1.3 should be compared with the decompositions of decorated Riemann surfaces studied in [3]. A decoration of a finite volume, cusped hyperbolic surface $X$ is a subset $B \subset X$ consisting of disjoint and simple horocycles about each cusp of $X$. Associated to each decoration of $X$ is a

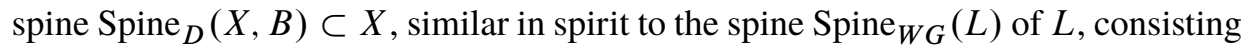
of all points in $X$ which have multiple shortest paths to $B$. In fact, for finite volume $L$, one can show that the well-girthed spine $\operatorname{Spine}_{W G}(L)$ is associated to a particular decoration of $L$.

Algorithm. Combining Theorem 1.2 with standard methods for traversing graphs (e.g. breadth-first search) gives an algorithm for exploring $L$ and enumerating elements of $\operatorname{PSL}\left(X_{0}, q_{0}\right)$. Breadth-first search (BFS) proceeds by iteratively enlarging a list of "known" vertices and edges by, at each stage, finding the neighbors of all of the previously known vertices.

The key ingredients to implementing BFS are methods to (1) list the neighbors of a particular vertex, and (2) determine whether a particular neighbor is among the list

${ }^{1}$ If $L$ has orbifold points, we mean that there is a homotopy $h_{t}: L \rightarrow L$ defined on the coarse space associated to $L$ and a homotopy $\tilde{h}_{t}: \tilde{L} \rightarrow \tilde{L}$ defined on a good cover $\pi: \tilde{L} \rightarrow L$ so that $\tilde{h}_{0}$ is the identity, $\tilde{h}_{t}$ restricts to the identity on $\pi^{-1}\left(\operatorname{Spine}_{W G}(L)\right)$ and $\tilde{h}_{1}$ is a retraction onto $\pi^{-1}\left(\operatorname{Spine}_{W G}(L)\right)$ and $\pi \circ \tilde{h}_{t}=h_{t} \circ \pi$. 
of known vertices. The vertices of $\operatorname{Spine}_{W G}(L)$ are points $[X, q] \in V=\bigcup_{k \geq 3} L[k]$ and the valence of $[X, q]$ is typically $\# N(X, q)$. It is straightforward to compute the neighbors of $[X, q]$ from the saddle periods of $(X, q)$, and we describe an algorithm to do so at the end of Section 3 (see also Figure 2). To check whether a particular neighbor of $[X, q]$ is among the previously known vertices, we compare its Delaunay triangulation with those of the known vertices. In this way, we enumerate the vertices, edges and cycles on $\operatorname{Spine}_{W G}(L)$ and compute elements on $\operatorname{PSL}\left(X_{0}, q_{0}\right)$.

$$
\mathbf{V}=\left\{\begin{array}{c}
-11+\sqrt{11},-\frac{11}{2},-\sqrt{11}, \frac{1}{3}(-11+\sqrt{11}), \frac{1}{7}(-11-2 \sqrt{11}), \frac{4}{7}(-11+2 \sqrt{11}), \frac{1}{5}(-22+3 \sqrt{11}), \\
\frac{3}{10}(-11+\sqrt{11}),-2, \frac{1}{5}(-33+7 \sqrt{11}), \frac{1}{4}(-11+\sqrt{11}), \frac{1}{7}(-22+3 \sqrt{11}), \frac{1}{5}(-11+\sqrt{11}), 0, \frac{1}{5}(11-\sqrt{11}), \\
\frac{1}{4}(11-\sqrt{11}), \frac{1}{5}(33-7 \sqrt{11}), 2,-\frac{3}{10}(-11+\sqrt{11}), \frac{1}{5}(22-3 \sqrt{11}), \frac{1}{3}(11-\sqrt{11}), \sqrt{11}, \infty
\end{array}\right\} .
$$
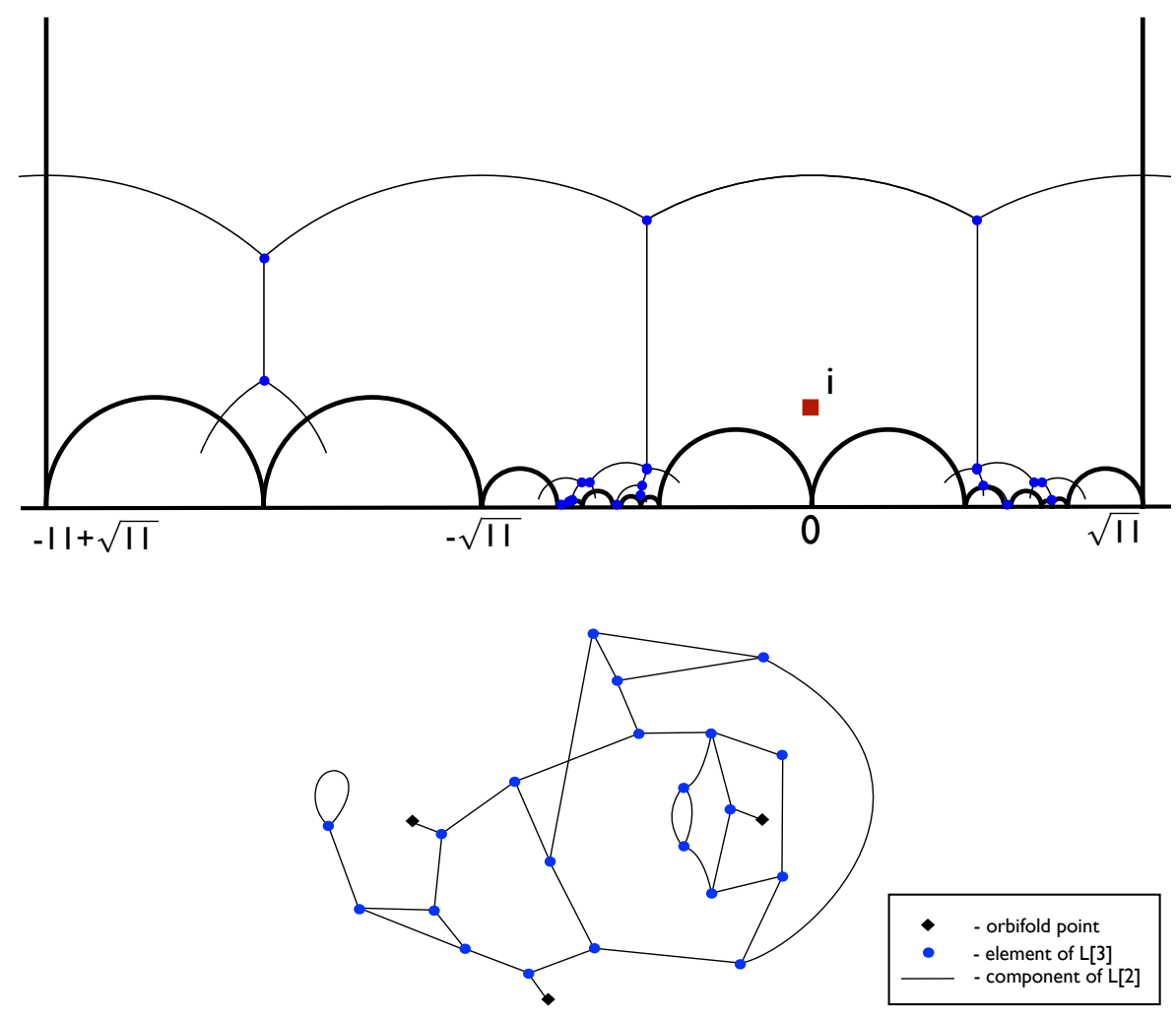

Figure 1. The Riemann surface $L_{44} \cong \mathbb{H} / \operatorname{PSL}\left(X_{44}, q_{44}\right)$ of genus one has nine cusps and three orbifold points of order two. The convex hull of $V \subset \partial \mathbb{H}$ (top) is an ideal 23-gon (middle) and a fundamental domain for $\operatorname{PSL}\left(X_{44}, q_{44}\right)$. Our algorithm computes a fundamental domain and generators for $\operatorname{PSL}\left(X_{44}, q_{44}\right)$ by performing a breadth first search on the well-girthed spine Spine $_{W G}\left(L_{44}\right)$ (bottom). 
By Theorem 1.3, when $\operatorname{PSL}\left(X_{0}, q_{0}\right)$ is a lattice, the well-girthed spine has finitely many vertices and edges. The BFS will terminate yielding a complete list of vertices and edges for $\operatorname{Spine}_{W G}(L)$ from which one can compute a fundamental domain and set of generators for $\operatorname{PSL}\left(X_{0}, q_{0}\right)$. In Section 4 we apply our algorithm to several examples of Veech groups of genus two quadratic differentials (see also Figures 1 and 4).

Proof of Theorem 1.1. As described in Section 4, the pair $\left(S_{D}, \sigma_{D}\right)$ listed in Table 1 encodes an ideal hyperbolic polygon $D\left(S_{D}\right)$ and generators $\Gamma_{\left(S_{D}, \sigma_{D}\right)} \subset G$ for a Fuchsian group $\Gamma_{D}$ with fundamental domain $D\left(S_{D}\right)$. By comparing Delaunay triangulations as described above, we check that each element of $\Gamma_{\left(S_{D}, \sigma_{D}\right)}$ is in $\operatorname{PSL}\left(X_{D}, q_{D}\right)$ and conclude that $\Gamma_{D}$ is finite index in $\operatorname{PSL}\left(X_{D}, q_{D}\right)$.

To check that $\operatorname{PSL}\left(X_{D}, q_{D}\right)$ is no larger, we compute $\operatorname{Spine}_{W G}\left(\mathbb{H} / \operatorname{PSL}\left(X_{D}, q_{D}\right)\right)$ via the process outlined in the previous paragraph. Since $\mathbb{H} / \operatorname{PSL}\left(X_{D}, q_{D}\right)$

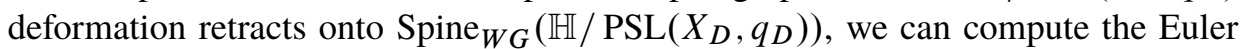
characteristic and hyperbolic area of $\mathbb{H} / \operatorname{PSL}\left(X_{D}, q_{D}\right)$ from the isomorphism type of the well-girthed spine. Checking that the area of $D\left(S_{D}\right)$ is equal to the area of $\mathbb{H} / \operatorname{PSL}\left(X_{D}, q_{D}\right)$ ensures that $\Gamma_{D}=\operatorname{PSL}\left(X_{D}, q_{D}\right)$.

Other algorithms. There are several other methods for computing elements in $\operatorname{PSL}\left(X_{0}, q_{0}\right)$. The algorithm described in [10] searches for parabolic elements in $\operatorname{PSL}\left(X_{0}, q_{0}\right)$ and will find generators for lattice Veech groups only if the quotient $\mathbb{H} / \operatorname{PSL}\left(X_{0}, q_{0}\right)$ has genus zero. A stratification of $L$ by hyperbolic polygons based on Delaunay triangulations is studied in [19] and an algorithm based on this stratification is suggested in [4]. While this algorithm will theoretically give generators and a fundamental domain for arbitrary Veech groups, the stratification of $L$ in Theorem 1.2 will typically be much simpler than the Delaunay stratification and Theorem 1.1 is the first example of an explicit computation of fundamental domains and generators for non-arithmetic Veech groups with higher genus quotients. An algorithm for finding generators of $\operatorname{PSL}\left(X_{0}, q_{0}\right)$ when $\operatorname{PSL}\left(X_{0}, q_{0}\right)$ is arithmetic, i.e. commensurable to $\mathrm{PSL}_{2}(\mathbb{Z})$, is given in [15], and in [6] it is shown that every finite index subgroup of $\Gamma(2)=\operatorname{ker}\left(\mathrm{PSL}_{2}(\mathbb{Z}) \rightarrow \mathrm{PSL}_{2}(\mathbb{Z} / 2 \mathbb{Z})\right)$ is a Veech group.

Notes and references. The spine $\operatorname{Spine}_{W G}(L)$ is also studied in [16] to give a characterization of surfaces with lattice Veech groups. In particular, the retraction $L \rightarrow$ Spine $_{W G}(L)$ we define in Proposition 3.14 also appears there (cf. proof of Proposition 4.2 in [16]). Theorem 1.3 can be read as a converse to Corollary 4.5 of [16] which shows that if $L[2]$ has finitely many components then $L$ has finite volume.

Our terminology is meant to suggest a comparison with well-rounded lattices, which form a spine for the homogeneous space $\mathrm{SL}_{n}(\mathbb{R}) / \mathrm{SL}_{n}(\mathbb{Z})$ [1]. For examples of quadratic differentials with complicated (and not necessarily lattice) Veech groups, 
see e.g. $[11,17]$. It would be interesting to explore those Veech groups using our algorithm. For further background on the moduli space of quadratic differentials $Q \mathcal{M}_{g}$, the $G$-action on $Q \mathcal{M}_{g}$ and Veech groups, see e.g. [9] or [20].

Acknowledgements. I would like to thank C. McMullen and S. Kerckhoff for useful conversations and the referee for helpful suggestions. I would also like to thank C. McMullen for sharing computer code that enumerates the saddle periods on $\left(X_{0}, q_{0}\right)$ on which our algorithm and the computations appearing in this paper rely. The author was supported in part by National Science Foundation grant DMS-1103654.

\section{Quadratic differentials}

In this section, we collect background and fix notation about quadratic differentials and the $G$-action on $Q \mathcal{M}_{g}$. For further background, see $[9,20]$.

Quadratic differentials. A holomorphic quadratic differential on a Riemann surface $X \in \mathcal{M}_{g}$ is a holomorphic section of the square of the cotangent bundle on $X$. The collection $Q(X)$ of all holomorphic quadratic differentials on $X$ forms a $(3 g-3)$-dimensional complex vector space and, as $X$ ranges in $\mathcal{M}_{g}$, the non-zero elements in $Q(X)$ form a bundle $Q \mathcal{M}_{g} \rightarrow \mathcal{M}_{g}$. A non-zero differential $q \in Q(X)$ gives a metric $|q|$ on $X$ which is flat except for cone singularities at the zeros $Z(q)$ of $q$. The differential $q$ also determines a foliation $\mathcal{F}(q)$ of $X \backslash Z(q)$ by horizontal geodesics.

A typical way to specify a pair $(X, q)$ is to glue parallel sides of equal length on a collection of polygons $\left\{P_{1}, \ldots, P_{k}\right\}$ in $\mathbb{C}$ by transition functions of the form $z \mapsto \pm z+c$ (see Section 4 for examples):

$$
(X, q)=\bigcup_{i}\left(P_{i}, d z^{2}\right) / \sim
$$

Lines in $\mathbb{C}$ give geodesics on $X$ and horizontal lines give leaves of $\mathcal{F}(q)$. The quadratic differential up to scale represented by $(X, q)$ will be denoted by $[X, q]$.

Saddle connections and saddle periods. A saddle connection on $(X, q)$ is a $|q|$ geodesic $\gamma:[a, b] \rightarrow X$ beginning and ending at $Z(q)$ and avoiding $Z(q)$ otherwise. Along $\gamma$ there are two choices of square roots for $q$ and the complex numbers $\int_{\gamma} \pm \sqrt{q}$ are called periods of $\gamma$. The image in $\mathbb{C}^{*} /(z \sim-z)$ of the set of all saddle periods on $(X, q)$ will be denoted by:

$$
\operatorname{Per}(X, q)=\left\{\int_{\gamma} \pm \sqrt{q}: \gamma \text { is a saddle connection on }(X, q)\right\} \subset \mathbb{C}^{*} /(z \sim-z) .
$$


Note that $\operatorname{Per}\left(X, \lambda^{2} q\right)=\{ \pm \lambda v: \pm v \in \operatorname{Per}(X, q)\}=\lambda \operatorname{Per}(X, q)$. The set $\operatorname{Per}(X, q)$ is discrete and its preimage in $\mathbb{C}$ is bounded away from zero.

The set of all shortest saddle periods in $\operatorname{Per}(X, q)$ will be denoted by:

$$
\operatorname{Per}_{m}(X, q)=\{ \pm v \in \operatorname{Per}(X, q):| \pm v| \leq| \pm w| \text { for any } \pm w \in \operatorname{Per}(X, q)\} .
$$

As in Section 1, we will call $(X, q)$ a girth differential if one of the shortest saddle periods on $(X, q)$ is horizontal of length one, i.e. $\pm 1 \in \operatorname{Per}_{m}(X, q)$. Since $\operatorname{Per}_{m}(X, q)$ is a discrete subset of a circle, it is a non-empty, cyclically ordered and finite set. The sets $N(X, q)$ and $\operatorname{Per}_{m}(X, q)$ are in natural bijection:

Proposition 2.1. The map $\pm \lambda \mapsto \lambda^{-2}$ gives a bijection between $\operatorname{Per}_{m}(X, q)$ and $N(X, q)$.

Proof. For any $\lambda \in \mathbb{C}^{*}$, the shortest saddle periods on $(X, q)$ and $\left(X, \lambda^{2} q\right)$ are related by $\lambda \cdot \operatorname{Per}_{m}(X, q)=\operatorname{Per}_{m}\left(X, \lambda^{2} q\right)$. One of the shortest saddle connections on $\left(X, \lambda^{-2} q\right)$ is horizontal and of length one if and only if $\pm \lambda \in \operatorname{Per}_{m}(X, q)$.

$\mathrm{GL}_{2}^{+}(\mathbb{R})$ - and $\boldsymbol{G}$-actions. Let $\mathrm{GL}_{2}^{+}(\mathbb{R})$ denote the group of two-by-two matrices with positive determinant and let $G$ be the quotient $\mathrm{GL}_{2}^{+}(\mathbb{R}) /\left\langle \pm\left(\begin{array}{ll}1 & 0 \\ 0 & 1\end{array}\right)\right\rangle$. The group $\mathrm{GL}_{2}^{+}(\mathbb{R})$ acts on $\mathbb{C}$ by real-linear maps:

$$
\left(\begin{array}{ll}
a & b \\
c & d
\end{array}\right) \cdot(x+i y)=(a x+b y)+(c x+d y) i .
$$

This action covers a $G$-action on the quotient $\mathbb{C}^{*} /(z \sim-z)$.

For a quadratic differential $(X, q)$ obtained by gluing together the polygons $P_{i} \subset \mathbb{C}$ and a matrix $A \in \mathrm{GL}_{2}^{+}(\mathbb{R})$, the quadratic differential $A \cdot(X, q)$ is obtained by gluing together the polygons $A \cdot P_{i}$ :

$$
A \cdot(X, q)=\bigcup_{i}\left(A \cdot P_{i}, d z^{2}\right) / \sim .
$$

The matrix $\left(\begin{array}{cc}-1 & 0 \\ 0 & -1\end{array}\right)$ stabilizes every point in $Q \mathcal{M}_{g}$, and this $\mathrm{GL}_{2}^{+}(\mathbb{R})$-action also covers a $G$-action. The Veech group of $(X, q)$ is its stabilizer in $G$ :

$$
\operatorname{PSL}(X, q)=\{A \in G: A \cdot(X, q)=(X, q)\} .
$$

An important property of saddle periods is that they are equivariant with respect to $G$, i.e.:

$$
\operatorname{Per}(A \cdot(X, q))=A \cdot \operatorname{Per}(X, q) .
$$

In particular, $\operatorname{PSL}(X, q)$ preserves the set $\operatorname{Per}(X, q)$. 
It is not true that the shortest saddle periods are equivariant with respect to $G$. Instead, we have:

Proposition 2.2. Fix any $(X, q) \in Q \mathcal{M}_{g}$, the set:

$$
U(X, q)=\left\{B \in G: \operatorname{Per}_{m}(B \cdot(X, q)) \subset B \operatorname{Per}_{m}(X, q)\right\}
$$

contains an open neighborhood of the identity.

Proof. The claim follows easily from the fact that $\operatorname{Per}(X, q)$ is discrete. Let $l_{0}$ be the length of one of the shortest saddle periods on $(X, q)$ and let $1 / 2>\epsilon>0$ be a number so that there are no saddle periods whose lengths belong to the interval $\left(l_{0},(1+\epsilon) l_{0}\right)$. We will show that $U(X, q)$ contains the image in $G$ of the set $U(\epsilon / 3)$ of matrices that distort lengths by a factor of at most $1+\epsilon / 3$ :

$U(\epsilon / 3)=\left\{g \in \mathrm{GL}_{2}^{+}(\mathbb{R}):(1+\epsilon / 3)^{-1}|v|<|g \cdot v|<(1+\epsilon / 3)|v|\right.$ for any $\left.v \in \mathbb{C}^{*}\right\}$.

If $g \in U(\epsilon / 3)$, then the length of the shortest saddle period on $g \cdot(X, q)$ is at most $(1+\epsilon / 3) l_{0}$ and the longest saddle period in $g^{-1} \cdot \operatorname{Per}_{m}(g \cdot(X, q)) \subset \operatorname{Per}(X, q)$ has length at most $(1+\epsilon / 3)^{2} l_{0}<(1+\epsilon) l_{0}$. Based on our choice of $\epsilon, \operatorname{Per}_{m}(g \cdot(X, q)) \subset$ $g \cdot \operatorname{Per}_{m}(X, q)$ and the image of $g$ in $G$ is in $U(X, q)$.

Proposition 2.2 yields the following as a corollary.

Proposition 2.3. For any $k \geq 1$, the set $\bigcup_{n \geq k} L[n]$ is closed in $L$.

Proof. We will show that the complement $V=\bigcup_{n<k} L[n]$ of $\bigcup_{n \geq k} L[n]$ is open. If $[X, q] \in V$, then $\operatorname{Per}_{m}(X, q)$ contains fewer than $k$ saddle periods. By Proposition 2.2, there is a neighborhood $U$ of the identity in $G$ consisting of matrices $g$ with $\operatorname{Per}_{m}(g \cdot(X, q)) \subset g \cdot \operatorname{Per}_{m}(X, q)$. The image of $U$ in $L$ under $g \mapsto[g \cdot(X, q)]$ is a neighborhood of $[X, q]$ and contained in $V$, so $V$ is open.

Euclidean similarities and the hyperbolic plane. We will denote by $\mathbb{C}^{*} \subset \mathrm{GL}_{2}^{+}(\mathbb{R})$ the subgroup of Euclidean similarities, i.e. the subgroup commuting with $S O(2)$. We will denote by $[A]$ the coset representative of $A$ in $\mathbb{C}^{*} \backslash \mathrm{GL}_{2}^{+}(\mathbb{R})$. We will identify the quotient $\mathbb{C}^{*} \backslash \mathrm{GL}_{2}^{+}(\mathbb{R})$ with the hyperbolic plane via the bijection $\mathbb{H} \rightarrow \mathbb{C}^{*} \backslash \mathrm{GL}_{2}^{+}(\mathbb{R})$ defined by

$$
\tau \mapsto\left[A_{\tau}\right] \text { where } A_{\tau}=\left(\begin{array}{ll}
1 & \operatorname{Re} \tau \\
0 & \operatorname{Im} \tau
\end{array}\right) .
$$

In particular, there is a unique holomorphic structure and metric on $\mathbb{C}^{*} \backslash \mathrm{GL}_{2}^{+}(\mathbb{R})$ for which this identification is an isometry. The map $f: \mathbb{H} \rightarrow L$ defined by $f([A])=\left[A \cdot\left(X_{0}, q_{0}\right)\right]$ is the universal covering map for $L$. 
Möbius transformations. The quotient $\mathbb{C}^{*} \backslash \mathrm{GL}_{2}^{+}(\mathbb{R})$ has a right $\mathrm{GL}_{2}^{+}(\mathbb{R})$-action (and $G$-action), with $B$ acting by $[A] \mapsto[A B]$. There is a unique Möbius transformation $m_{B}: \mathbb{H} \rightarrow \mathbb{H}$ with the property that $\left[A_{\tau} B\right]=\left[A_{m_{B}(\tau)}\right]$. Namely, if $B=\left(\begin{array}{ll}a & b \\ c & d\end{array}\right)$, then $m_{B}(\tau)=\frac{a \tau-b}{-c \tau+d}$. The universal cover $f: \mathbb{H} \rightarrow L$ satisfies $f \circ m_{B}=f$ for each $B \in \operatorname{PSL}\left(X_{0}, q_{0}\right)$ and $f$ covers an isometry $\mathbb{H} / \operatorname{PSL}\left(X_{0}, q_{0}\right) \rightarrow L$, where $B \in \operatorname{PSL}\left(X_{0}, q_{0}\right)$ acts on the right on $\mathbb{H}$ by $m_{B}$.

Geodesics, half planes and horoballs. We now state some facts about geodesics, half planes and horoballs in $\mathbb{H}=\mathbb{C}^{*} \backslash \mathrm{GL}_{2}^{+}(\mathbb{R})$ that are elementary to verify.

For any pair of distinct points $\pm v$ and $\pm w$ in $\mathbb{C}^{*} /(z \sim-z)$ with $v$ and $w$ linearly independent over $\mathbb{R}$, the set:

$$
\gamma( \pm v, \pm w)=\{[A] \in \mathbb{H}:|A \cdot( \pm v)|=|A \cdot( \pm w)|\}
$$

is the geodesic with endpoints $\frac{\operatorname{Re}(v)-\operatorname{Re}(w)}{\operatorname{Im}(w)-\operatorname{Im}(v)}$ and $-\frac{\operatorname{Re}(v)+\operatorname{Re}(w)}{\operatorname{Im}(v)+\operatorname{Im}(w)}$. For such $\pm v$ and $\pm w$, the set:

$$
T( \pm v, \pm w)=\{[A] \in \mathbb{H}:|A \cdot( \pm v)|<|A \cdot( \pm w)|\}
$$

is an open half plane with boundary $\partial T( \pm v, \pm w)=\gamma( \pm v, \pm w)$. Note if $v=\lambda w$ with $\lambda>1$ real, the set $\gamma( \pm v, \pm w)$ is empty, $T( \pm v, \pm w)$ is empty and $T( \pm w, \pm v)=\mathbb{H}$.

For any $\pm v \in \mathbb{C}^{*} /(z \sim-z)$ and real number $C>0$, the set:

$$
B( \pm v, C)=\left\{[A] \in \mathbb{H}: \frac{|A \cdot( \pm v)|^{2}}{\operatorname{det}(A)} \leq C\right\} .
$$

is the closed horoball tangent to $\partial \mathbb{H}$ at $-\operatorname{Re}(v) / \operatorname{Im}(v)$ and of radius $\frac{C}{2(\operatorname{Im} v)^{2}}$.

\section{Girth differentials and saddle periods}

In this section, we will prove Theorems 1.2 and 1.3 by studying the set $\operatorname{Per}\left(X_{0}, q_{0}\right)$ of saddle periods on $\left(X_{0}, q_{0}\right)$ and the action of $\operatorname{PSL}\left(X_{0}, q_{0}\right)$ on $\operatorname{Per}\left(X_{0}, q_{0}\right)$. We will associate to each finite subset $S \subset \operatorname{Per}\left(X_{0}, q_{0}\right)$ a set:

$$
H_{m}(S)=\left\{[A] \in \mathbb{H}: \operatorname{Per}_{m}\left(A \cdot\left(X_{0}, q_{0}\right)\right)=A \cdot S\right\} .
$$

We will characterize when $H_{m}(S)$ is non-empty and then prove the following proposition, allowing us to establish the claims about the components of $L[n]$ made in Theorem 1.2:

Proposition 3.1. Fix $S \subset \operatorname{Per}\left(X_{0}, q_{0}\right)$ with $H_{m}(S)$ non-empty. One of the following holds:

(1) $H_{m}(S)$ is an open, convex subset of $\mathbb{H}$ and contained in a horoball $(\# S=1)$; 
(2) $H_{m}(S)$ is an open geodesic arc of finite length $(\# S=2)$; or

(3) $H_{m}(S)$ is a single point $(\# S \geq 3)$.

The image of $H_{m}(S)$ under $[A] \mapsto\left[A \cdot\left(X_{0}, q_{0}\right)\right]$ is a connected component of $L[\# S]$.

We will then construct an explicit homotopy inverse for the inclusion

$$
\text { Spine }_{W G}(L) \rightarrow L
$$

using the $G$-action on $Q L$ to complete the proof of Theorem 1.2. The key observation is that the set of girth differentials is closed under multiplication by matrices of the form $\left(\begin{array}{cc}1 & 0 \\ 0 & e^{2 t}\end{array}\right)$ for $t>0$ :

Proposition 3.2. If $(X, q) \in Q L$ is a girth differential and $t \geq 0$, then $\left(\begin{array}{cc}1 & 0 \\ 0 & e^{2 t}\end{array}\right) \cdot(X, q)$ is also a girth differential.

This gives a foliation of the set of girth differentials by intervals which project to geodesic rays in $L$. The map collapsing these rays to their endpoints gives the desired homotopy inverse.

Finally, we will conclude this section by turning to the case where $L$ has finite volume. Using the Veech dichotomy, we will show that $L[1]$ contains a horoball neighborhood of each cusp of $L$ to conclude that $\operatorname{Spine}_{W G}(L)$ is contained in compact set and prove Theorem 1.3.

Ellipses. To start, we will characterize when $H_{m}(S)$ is empty. By an ellipse (respectively circle) in $\mathbb{C}^{*} /(z \sim-z)$ we will mean the image of an ellipse (respectively circle) in $\mathbb{C}$ invariant under $z \mapsto-z$. We will say a finite subset $S \subset$ $\operatorname{Per}\left(X_{0}, q_{0}\right)$ is supported by the ellipse $E \subset \mathbb{C}^{*} /(z \sim-z)$ if $S=E \cap \operatorname{Per}\left(X_{0}, q_{0}\right)$ and the region bounded by $E$ contains no saddle periods. Equivalently, an ellipse $E$ containing $S$ supports $S$ if and only if $\operatorname{Per}_{m}\left(A \cdot\left(X_{0}, q_{0}\right)\right)=A \cdot S$ whenever $A \cdot E$ is a circle.

The subsets of $\operatorname{Per}\left(X_{0}, q_{0}\right)$ supported by ellipses are those for which $H_{m}(S)$ is non-empty:

Proposition 3.3. Fix a finite set $S \subset \operatorname{Per}\left(X_{0}, q_{0}\right)$. The set $H_{m}(S)$ is non-empty if and only if $S$ is supported by an ellipse.

Proof. First suppose $[A] \in H_{m}(S)$ and let $E \subset \mathbb{C}^{*} /(z \sim-z)$ be the circle passing through $\operatorname{Per}_{m}\left(A \cdot\left(X_{0}, q_{0}\right)\right)=A \cdot S$. The ellipse $A^{-1} \cdot E$ supports $S$. Conversely, suppose $E$ supports $S$ and let $A \in G$ be any matrix for which $A \cdot E$ is a circle. For such an $A, \operatorname{Per}_{m}\left(A \cdot\left(X_{0}, q_{0}\right)\right)=A \cdot S$ and $[A] \in H_{m}(S)$.

The collection of subsets of $\operatorname{Per}\left(X_{0}, q_{0}\right)$ supported by ellipses:

$\operatorname{Ell}\left(X_{0}, q_{0}\right)=\left\{S \subset \operatorname{Per}\left(X_{0}, q_{0}\right): S\right.$ is supported by an ellipse $\}$.

and the collections $\operatorname{Ell}\left(X_{0}, q_{0}\right)[n]=\left\{S \in \operatorname{Ell}\left(X_{0}, q_{0}\right): \# S=n\right\}$ will play an important role in what follows. 
Convex sets. We now associate to each $S \in \operatorname{Ell}\left(X_{0}, q_{0}\right)$ a complete convex set $H(S)$ in $\mathbb{H}$.

Proposition 3.4. For any $S \in \operatorname{Ell}\left(X_{0}, q_{0}\right)$, the set:

$$
H(S)=\{[A] \in \mathbb{H}: \text { The set } A \cdot S \text { lies on a circle }\}
$$

is a convex and complete subset of $\mathbb{H}$. More specifically, $H(S)=\mathbb{H}$ if $\# S=1$, $H(S)$ is a geodesic if $\# S=2$ and $H(S)$ is a point if $\# S \geq 3$.

Proof. First suppose $\# S=1$. For any $[A] \in \mathbb{H}, A \cdot S$ consists of a single point $\pm v \in \mathbb{C}^{*} /(z \sim-z)$ which lies on the circle of radius $| \pm v|$. If $\# S=2$, i.e. $S=$ $\{ \pm v, \pm w\}$, then $H(S)$ is the geodesic $\gamma( \pm v \pm w)$ defined in Section 2 (note that $v$ is not a real multiple of $w$ since $S$ is supported by an ellipse). Finally, suppose $\# S \geq 3$. The preimage of $S$ in $\mathbb{C}$ consists of at least six points and there is exactly one ellipse $E$ in $\mathbb{C}^{*} /(z \sim-z)$ containing $S$. The set $H(S)$ consists of the single point $[A] \in \mathbb{H}$ for which $A \cdot E$ is a circle.

Next we study the set $H_{m}(S)$ defined at the beginning of this section:

Proposition 3.5. For any $S \in \operatorname{Ell}\left(X_{0}, q_{0}\right)$, the set:

$$
H_{m}(S)=\left\{[A] \in \mathbb{H}: \operatorname{Per}_{m}(A \cdot(X, q))=A \cdot S\right\}
$$

is a non-empty, convex and open subset of $H(S)$ and is contained in the intersection of horoballs $\bigcap_{ \pm v \in S} B\left( \pm v, \frac{\operatorname{Area}\left(X_{0}, q_{0}\right)}{\pi}\right)$.

Proof. The set $H_{m}(S)$ is non-empty by Proposition 3.3.

We will now show that $H_{m}(S)$ is open in $H(S)$. Fix a point $[A] \in H_{m}(S)$. By Proposition 2.2, there is a neighborhood $U$ of the identity in $G$ with the property that:

$$
\operatorname{Per}_{m}\left(B A \cdot\left(X_{0}, q_{0}\right)\right) \subset B \cdot \operatorname{Per}_{m}\left(A \cdot\left(X_{0}, q_{0}\right)\right)=B A \cdot S \text { whenever } B \in U .
$$

The image of $U$ under $g: B \mapsto[B A]$ is an open neighborhood of $[A]$. For any $B \in U$ with $g(B)=[B A] \in H(S), \operatorname{Per}_{m}\left(B A \cdot\left(X_{0}, q_{0}\right)\right)$ is a subset of $B A \cdot S$, a set which lies on a circle. It follows that $\operatorname{Per}_{m}\left(B A \cdot\left(X_{0}, q_{0}\right)\right)=B A \cdot S,[B A] \in H_{m}(S)$ and $g(U) \cap H(S)$ is contained in $g(U) \cap H_{m}(S)$.

Next, we will show that $H_{m}(S)$ is convex. Recall that, for any $\pm v$ and $\pm w$ in $\mathbb{C}^{*} /(z \sim-z)$, the set $T( \pm v, \pm w)=\{[A] \in \mathbb{H}:|A \cdot( \pm v)|<|A \cdot( \pm w)|\}$ is convex (and usually an open half plane). The region $H_{m}(S)$ satisfies:

$$
H_{m}(S)=H(S) \cap\left(\bigcap_{\substack{ \pm w \in \operatorname{Per}\left(X_{0}, q_{0}\right), \pm w \notin S, \pm v \in S}} T( \pm v, \pm w)\right),
$$

and is also convex. 
We will now show $H_{m}(S)$ is contained in the closed horoball $B\left( \pm v, \frac{\operatorname{Area}\left(X_{0}, q_{0}\right)}{\pi}\right)$ for each $\pm v \in S$. If $A \cdot S=\operatorname{Per}_{m}\left(A \cdot\left(X_{0}, q_{0}\right)\right)$, then the injectivity radius of the metric flat metric on $A \cdot\left(X_{0}, q_{0}\right)$ is at least $|A \cdot( \pm v)|$ for each $\pm v \in S$. Whenever $[A] \notin B\left( \pm v, \operatorname{Area}\left(X_{0}, q_{0}\right) / \pi\right)$, the area of $A \cdot\left(X_{0}, q_{0}\right)$ is small enough the injectivity radius of the flat metric is smaller than $|A \cdot( \pm v)|$.

A stratification of $\mathbb{H}$. We now show that the sets $H_{m}(S)$ stratify $\mathbb{H}$ and that the image of $H_{m}(S)$ under the universal covering map $f: \mathbb{H} \rightarrow L$ is an open subset of $L[\# S]$.

Proposition 3.6. The sets $H_{m}(S)$ as $S$ ranges in $\operatorname{Ell}\left(X_{0}, q_{0}\right)$ are pairwise disjoint and cover $\mathbb{H}$.

Proof. First suppose $H_{m}\left(S_{1}\right)$ and $H_{m}\left(S_{2}\right)$ intersect. If $[A] \in H_{m}\left(S_{1}\right) \cap H_{m}\left(S_{2}\right)$, then $A \cdot S_{1}=\operatorname{Per}_{m}\left(A \cdot\left(X_{0}, q_{0}\right)\right)=A \cdot S_{2}$ and, since $A$ is invertible, $S_{1}=S_{2}$. To see that the sets $H_{m}(S)$ cover $\mathbb{H}$, note that $[A]$ is in $H_{m}(S)$ where $S=A^{-1} \cdot \operatorname{Per}_{m}(A$. $\left.\left(X_{0}, q_{0}\right)\right)$.

Proposition 3.7. For any $S \in \operatorname{Ell}\left(X_{0}, q_{0}\right)[n]$, the image $f\left(H_{m}(S)\right)$ is an open subset of $L[n]$.

Proof. The set $f\left(H_{m}(S)\right)$ is contained in $L[n]$ since, for any $[A] \in H_{m}(S), f([A])=$ $\left[A \cdot\left(X_{0}, q_{0}\right)\right]$ has:

$$
\# N\left(A \cdot\left(X_{0}, q_{0}\right)\right)=\# \operatorname{Per}_{m}\left(A \cdot\left(X_{0}, q_{0}\right)\right)=\# A \cdot S=n .
$$

Now fix any $[A] \in H_{m}(S)$. We will show that $f\left(H_{m}(S)\right)$ contains a neighborhood of $f([A])$ in $L[n]$. By Proposition 2.2, there is a neighborhood $U$ of the identity in $G$ with the property that $\operatorname{Per}_{m}\left(B A \cdot\left(X_{0}, q_{0}\right)\right) \subset B \operatorname{Per}_{m}\left(A \cdot\left(X_{0}, q_{0}\right)\right)$ whenever $B \in U$. The image of $U$ under $g: B \mapsto\left[B A \cdot\left(X_{0}, q_{0}\right)\right]$ is an open neighborhood of $f([A])$ in $L$, and $g(B)$ is in $L[n]$ if and only if:

$$
\operatorname{Per}_{m}\left(B A \cdot\left(X_{0}, q_{0}\right)\right)=B \operatorname{Per}_{m}\left(A \cdot\left(X_{0}, q_{0}\right)\right)=B A \cdot S,
$$

i.e. $[B A] \in H_{m}(S)$. The neighborhood $g(U) \cap L[n]$ of $f([A])$ is contained in $f\left(H_{m}(S)\right)$.

Veech group. We now study the action of $\operatorname{PSL}\left(X_{0}, q_{0}\right)$ on $\mathbb{H}$. We start by showing that $\operatorname{PSL}\left(X_{0}, q_{0}\right)$ permutes the pieces of the stratification of $\mathbb{H}=\bigcup_{S \in \operatorname{Ell}\left(X_{0}, q_{0}\right)} H_{m}(S)$ :

Proposition 3.8. Fix $B \in \operatorname{PSL}\left(X_{0}, q_{0}\right)$ and $S \in \operatorname{Ell}\left(X_{0}, q_{0}\right)$. The set $B^{-1} \cdot S$ is in $\operatorname{Ell}\left(X_{0}, q_{0}\right)$ and the Möbius transformation $m_{B}:[A] \mapsto[A B]$ restricts to an isometry between $H_{m}(S)$ and $H_{m}\left(B^{-1} \cdot S\right)$. 
Proof. Fix $[A] \in H_{m}(S)$, i.e. $\operatorname{Per}_{m}\left(A \cdot\left(X_{0}, q_{0}\right)\right)=A \cdot S$. If $E$ is any ellipse supporting $S$, then $B^{-1} \cdot E$ supports $B^{-1} \cdot S$. The coset $[A B]=m_{B}([A])$ is in $H_{m}\left(B^{-1} S\right)$ since:

$$
\operatorname{Per}_{m}\left(A B \cdot\left(X_{0}, q_{0}\right)\right)=\operatorname{Per}_{m}\left(A \cdot\left(X_{0}, q_{0}\right)\right)=A \cdot S=(A B) \cdot\left(B^{-1} \cdot S\right) .
$$

This shows that $m_{B}$ sends $H_{m}(S)$ into $H_{m}\left(B^{-1} \cdot S\right)$. Since $m_{B^{-1}}=m_{B}^{-1}, m_{B}$ restricts to an isometry between $H_{m}(S)$ and $H_{m}\left(B^{-1} \cdot S\right)$.

Partitions of $\boldsymbol{L}$ and $\boldsymbol{L}[\boldsymbol{n}]$. By Proposition 3.8, the Veech group permutes the components of the stratification $\mathbb{H}=\bigcup_{S \in \operatorname{Ell}\left(X_{0}, q_{0}\right)} H_{m}(S)$, giving a stratification of the quotient $\mathbb{H} / \operatorname{PSL}\left(X_{0}, q_{0}\right)$ into disjoint sets:

$$
\mathbb{H} / \operatorname{PSL}\left(X_{0}, q_{0}\right)=\bigcup_{[S] \in \operatorname{Ell}\left(X_{0}, q_{0}\right) / \operatorname{PSL}\left(X_{0}, q_{0}\right)} H_{m}(S) / \operatorname{Stab}(S) .
$$

Here $[S]$ is the coset in $\operatorname{Ell}\left(X_{0}, q_{0}\right) / \operatorname{PSL}\left(X_{0}, q_{0}\right)$ containing $S$ and $\operatorname{Stab}(S)$ is the stabilizer of $S$ in $\operatorname{PSL}\left(X_{0}, q_{0}\right)$. Since the universal covering map $f: \mathbb{H} \rightarrow L$ factors through an isometry $\mathbb{H} / \operatorname{PSL}\left(X_{0}, q_{0}\right) \rightarrow L$ and $f\left(H_{m}(S)\right)$ is contained in $L[\# S]$, this stratification of $\mathbb{H} / \operatorname{PSL}\left(X_{0}, q_{0}\right)$ gives a stratification of $L[n]$ into disjoint sets:

$$
\begin{aligned}
& L[n]=\bigcup_{[S] \in \operatorname{Ell}\left(X_{0}, q_{0}\right)[n] / \operatorname{PSL}\left(X_{0}, q_{0}\right)} f\left(H_{m}(S)\right) .
\end{aligned}
$$

By Proposition 3.6, $f\left(H_{m}(S)\right)$ is open in $L[n]$, giving:

Proposition 3.9. For each $S \in \operatorname{Ell}\left(X_{0}, q_{0}\right)[n]$, the set $f\left(H_{m}(S)\right)$ is a connected component of $L[n]$. The map $[S] \mapsto f\left(H_{m}(S)\right)$ gives a bijection between the connected components of $L[n]$ and $\operatorname{Ell}\left(X_{0}, q_{0}\right)[n] / \operatorname{PSL}\left(X_{0}, q_{0}\right)$.

Proof. The sets $f\left(H_{m}(S)\right)$ as $S$ ranges over coset representatives in $\operatorname{Ell}\left(X_{0}, q_{0}\right)[n] /$ $\operatorname{PSL}\left(X_{0}, q_{0}\right)$ are disjoint and open subsets of $L[n]$. They are also connected since $H_{m}(S)$ is convex (and therefore path connected).

We are now ready to prove Proposition 3.1 stated at the beginning of this section:

Proof of Proposition 3.1. Fix $S \subset \operatorname{Per}\left(X_{0}, q_{0}\right)$ with $H_{m}(S)$ not empty, i.e. $S \in$ Ell $\left(X_{0}, q_{0}\right)$. In Proposition 3.9 we showed that the image of $H_{m}(S)$ under $[A] \mapsto$ $\left[A \cdot\left(X_{0}, q_{0}\right)\right]$ is a connected component of $L[n]$.

The remaining claims about $H_{m}(S)$ follow from Propositions 3.4 and 3.5. If $\# S=1, H_{m}(S)$ is an convex, open subset of $H(S)=\mathbb{H}$ and contained in the horoball $B\left( \pm v\right.$, Area $\left.\left(X_{0}, q_{0}\right) / \pi\right)$ where $\pm v \in S$. If $\# S=2, H_{m}(S)$ is an open and convex subset of the geodesic $H(S)$ and is contained in the intersection $\bigcap_{ \pm v \in S} B\left( \pm v, \frac{\operatorname{Area}\left(X_{0}, q_{0}\right)}{\pi}\right)$ which is compact. Such a set must be an open geodesic arc of finite length. Finally, if $\# S \geq 3$, then $H_{m}(S)$ is a point. 
Connected components of $L[n]$. We are now ready to prove the claims in Theorem 1.2 about the connected components of $L[n]$. We start with $L[1]$ :

Proposition 3.10. Each connected component of $L[1]$ is diffeomorphic to the disk or the annulus.

Proof. By Proposition 3.9, each connected component of $L[1]$ is equal to $f\left(H_{m}(S)\right)$ for some $S \in \operatorname{Ell}\left(X_{0}, q_{0}\right)[1]$. By Proposition 3.8, the quotient $H_{m}(S) / \operatorname{Stab}(S)$ injects into the quotient $\mathbb{H} / \operatorname{PSL}\left(X_{0}, q_{0}\right)$, and the restriction of $f$ to $H_{m}(S)$ covers a bijection from the quotient $H_{m}(S) / \operatorname{Stab}(S)$ onto $f\left(H_{m}(S)\right)$. This bijection is a diffeomorphism since it is a local isometry.

We need to show that $H_{m}(S) / \operatorname{Stab}(S)$ is diffeomorphic to the disk or the annulus. Since $H_{m}(S)$ is a convex open set in $H(S)=\mathbb{H}, H_{m}(S)$ is diffeomorphic to a disk. The stabilizer $\operatorname{Stab}(S)$ is a unipotent subgroup of the discrete group $\operatorname{PSL}\left(X_{0}, q_{0}\right)$. Either $\operatorname{Stab}(S)$ is trivial and $f\left(H_{m}(S)\right)$ is diffeomorphic to the disk, or $\operatorname{Stab}(S)$ is isomorphic to $\mathbb{Z}$, acts freely and properly discontinuously on $H_{m}(S)$ and $H_{m}(S)$ is diffeomorphic to the annulus.

Proposition 3.11. Each connected component of L[2] is a finite length geodesic arc.

Proof. By Proposition 3.9, each connected component of $L$ [2] is equal to $f\left(H_{m}(S)\right)$ for some $S \in \operatorname{Ell}\left(X_{0}, q_{0}\right)$ [2]. By Proposition 3.5, $H_{m}(S)$ is an open convex subset of the geodesic $H(S)$ and is contained in the intersection of a pair of horoballs tangent to distinct points in $\partial \mathbb{H}$. The set $H_{m}(S)$ is a finite length geodesic arc and so is its image $f\left(H_{m}(S)\right)$ in $L$.

Proposition 3.12. The set $\bigcup_{n \geq 3} L[n]$ is discrete in $L$.

Proof. Let $[A] \in \mathbb{H}$ be any point with $f([A])=\left[A \cdot\left(X_{0}, q_{0}\right)\right] \in L[n]$ for $n \geq 3$. Set $S=A^{-1} \operatorname{Per}_{m}\left(A \cdot\left(X_{0}, q_{0}\right)\right)$, so that $[A] \in H_{m}(S)$. By Proposition 2.2, there is a neighborhood $U$ of the identity in $G$ with the property that $\operatorname{Per}_{m}\left(B A \cdot\left(X_{0}, q_{0}\right)\right) \subset$ $B \cdot \operatorname{Per}_{m}\left(A \cdot\left(X_{0}, q_{0}\right)\right)=B A \cdot S$ whenever $B \in U$.

The image of $U$ under $g: B \mapsto f([B A])$ is an open set in $L$. Suppose $g(B) \in L[k]$ with $k \geq 3$, and choose $S^{\prime} \subset S$ so $\operatorname{Per}_{m}\left(B A \cdot\left(X_{0}, q_{0}\right)\right)=B A \cdot S^{\prime}$. Since $S^{\prime}$ contains at least three points, there is a unique ellipse $E$ supporting $S^{\prime}$. The matrix $B$ takes the circle $A \cdot E$ into the circle $B A \cdot E$ and is therefore a Euclidean similarity. In other words, the only point in $g(U) \cap \bigcup_{n \geq 3} L[n]$ is $f([A])$.

Retraction and homotopy equivalence. We now show that $L$ deformation retracts onto $\bigcup_{n \geq 2} L[n]$ by constructing an explicit homotopy inverse. We start by showing that $L[1]$ is foliated by geodesic rays. To do so, we will show that the set of girth differentials in $Q L$ :

$$
G L=\{(X, q) \in Q L:(X, q) \text { is a girth differential }\}
$$


is invariant under multiplication by $g_{t}=\left(\begin{array}{cc}1 & 0 \\ 0 & e^{2 t}\end{array}\right)$ for $t \geq 0$. The following Proposition implies Proposition 3.2:

Proposition 3.13. There is a continuous function $T: G L \rightarrow \mathbb{R}_{\leq 0}$ so that, for any $(X, q) \in G L$, we have:

(1) $g_{t} \cdot(X, q)$ is a girth differential and $\# N\left(g_{t} \cdot(X, q)\right)=1$ for $t>T(X, q)$;

(2) $g_{t} \cdot(X, q)$ is not a girth differential for $t<T(X, q)$; and

(3) $g_{t} \cdot(X, q)$ is a girth differential and \#N( $\left.g_{t} \cdot(X, q)\right) \geq 2$ for $t=T(X, q)$.

Proof. For any $\pm(x+i y) \in \operatorname{Per}(X, q)$ with $|x|<1$, the number $t_{0}=\frac{1}{4} \log \left(\frac{1-x^{2}}{y^{2}}\right)$ is non-positive since $x^{2}+y^{2}>1$. The number $t_{0}$ also has the property that $\left|\left(\begin{array}{cc}1 & 0 \\ 0 & e^{2 t}\end{array}\right) \cdot( \pm(x+i y))\right|$ is greater than one for $t>t_{0}$, equal to one when $t=t_{0}$ and is less than one for $t<t_{0}$. It is straightforward to verify that the function $T(X, q)$ given explicitly by:

$$
T(X, q)=\sup _{\substack{ \pm(x+i y) \in \operatorname{Per}(X, q) \\|x|<1}} \frac{1}{4} \log \left(\frac{1-x^{2}}{y^{2}}\right) .
$$

satisfies the desired properties. Note that the intersection of $\operatorname{Per}(X, q)$ with the strip $\{x+i y:|x|<1\}$ is non-empty since the area of $|q|$ is finite.

Proposition 3.13 shows that $L[1]$ is foliated by open half-infinite geodesic rays whose endpoints lie in $\bigcup_{n \geq 2} L[n]$. The map sending $[X, q] \in L[1]$ to the endpoint of the leaf of this foliation gives a homotopy inverse for the inclusion $\bigcup_{n \geq 2} L[n]$ :

Proposition 3.14. The inclusion $\operatorname{Spine}_{W G}(L) \rightarrow L$ is a homotopy equivalence, with homotopy inverse:

$$
\bar{h}: L \rightarrow \operatorname{Spine}_{W G}(L)
$$

defined by $\bar{h}([X, q])=\left[g_{T(X, q)} \cdot(X, q)\right]$ whenever $(X, q) \in G L$.

Proof. For $(X, q) \in G L$ and $t \geq 0$ define:

$$
h_{t}(X, q)=g_{\max (-t, T(X, q))} \cdot(X, q) .
$$

The functions $h_{t}: G L \rightarrow G L$ are continuous and depend continuously on $t$. Also, $h_{t}$ covers a well defined map $\bar{h}_{t}: L \rightarrow L$ since, whenever $\mathbb{C}^{*} q$ contains more than one girth differential, $T\left(X, q_{i}\right)=0$ for each $i$. The function $\bar{h}_{0}$ is the identity map on $L$ and the function $\bar{h}_{t}$ restricts to the identity on $\operatorname{Spine}_{W G}(L)$ for every $t \geq 0$. As $t$ tends to infinity, $\bar{h}_{t}$ tends uniformly on compact sets to the retraction $\bar{h}_{\infty}=\bar{h}$.

The functions $\bar{h}_{t}$ gives the desired homotopy equivalence, at least between the coarse space associated to the orbifold $L$ and the subset $\bigcup_{n \geq 2} L[n]$. If $L$ has orbifold points, we can replace $L$ by a good $\operatorname{cover} \pi: \tilde{L} \rightarrow L$, say by marking the $\mathbb{Z} / n \mathbb{Z}$-homology of $[X, q] \in L$ for $n$ large enough. It is straightforward to define a 
homotopy $\tilde{h}_{t}$ between the identity on $\tilde{L}$ and a retraction onto $\pi^{-1}\left(\bigcup_{n \geq 2} L[2]\right)$ which satisfies $\pi \circ \tilde{h}_{t}=h_{t} \circ \pi$. Note that the orbifold points of $L$ all lie in $\bigcup_{n \geq 2} L[n]$ since the orbifold order of $[X, q] \in L[n]$ divides $n$.

Proof of Theorem 1.2. The sets $L[n]$ are clearly disjoint and give a stratification $L=\bigcup_{n \geq 1} L[n]$. The set $\bigcup_{n \geq k} L[n]$ for each $k$ is closed by Proposition 2.3. The other claims about the components of $L[n]$ are established in Propositions 3.10, 3.11 and 3.12. That $\bigcup_{n \geq 2} L[n] \rightarrow L$ is a homotopy equivalence is established in Proposition 3.14 .

Lattice Veech groups. We now turn to the case when $\operatorname{PSL}\left(X_{0}, q_{0}\right)$ is a lattice and $L$ has finite volume.

Cusps. A cusp of a Fuchsian group $\Gamma$ is the fixed point $x \in \partial \mathbb{H}$ of any unipotent element $g \in \Gamma$. The set of all cusps of $\Gamma$ we will denote by $C(\Gamma)$. A cusp of the quotient $V=\mathbb{H} / \Gamma$ is a $\Gamma$-orbit in $C(\Gamma)$ and we will denote the set of all cusps on $V$ by $C(V)$. When the volume of $V$ is finite, the set $C(V)$ is finite and can be added to $V$ to give a closed surface $\bar{V}=V \cup C(V)$.

Cylinder decompositions. It is well known that if $\operatorname{PSL}\left(X_{0}, q_{0}\right)$ contains a unipotent element stabilizing the line $L=\mathbb{R} \cdot( \pm(x+i y))$ of slope $y / x$, then there is a collection $\left\{\gamma_{1}, \ldots, \gamma_{n}\right\}$ of saddle connections whose periods lie in $L$ and whose complement in $\left(X_{0}, q_{0}\right)$ is a disjoint union of metric cylinders $\left\{C_{1}, \ldots, C_{k}\right\}$. We will need the following partial converse for lattice Veech groups which is a consequence of the famous Veech dichotomy [18]:

Theorem 3.15 (Veech). Suppose $\operatorname{PSL}\left(X_{0}, q_{0}\right)$ is a lattice. Each saddle period $\pm v \in \operatorname{Per}\left(X_{0}, q_{0}\right)$ is parallel to a cylinder decomposition of $\left(X_{0}, q_{0}\right)$ and is stabilized by a unipotent element in $\operatorname{PSL}\left(X_{0}, q_{0}\right)$.

The Veech dichotomy allows us to prove:

Proposition 3.16. The following three subsets of $\partial \mathbb{H}$ are equal:

- $T_{1}=C\left(\operatorname{PSL}\left(X_{0}, q_{0}\right)\right)$,

- $T_{2}=\left\{-x / y \in \partial \mathbb{H}: \pm(x+i y) \in \operatorname{Per}\left(X_{0}, q_{0}\right)\right\}$, and

- $T_{3}=\left\{-x / y \in \partial \mathbb{H}:\{ \pm(x+i y)\} \in \operatorname{Ell}\left(X_{0}, q_{0}\right)\right\}$.

Proof. The sets $T_{1}$ and $T_{2}$ are equal by Theorem 3.15. The sets $T_{2}$ and $T_{3}$ are equal since a collection $S=\{ \pm v\} \subset \operatorname{Per}\left(X_{0}, q_{0}\right)$ of saddle periods consisting of a single saddle period is supported by an ellipse if and only if $\pm v$ is the shortest saddle period in the line $\mathbb{R} \cdot( \pm v)$.

Proposition 3.17. Suppose $L$ has finite volume, and $S=\{ \pm v\} \in \operatorname{Ell}\left(X_{0}, q_{0}\right)$. The set $H_{m}(S)$ contains $B( \pm v, K)$ for some $K>0$. 
Proof. When $L$ has finite volume, the complement in $\left(X_{0}, q_{0}\right)$ of the set of all saddle connections with periods parallel to $\pm v$ is a disjoint union of metric cylinders $\left\{C_{1}, \ldots C_{k}\right\}$. Let $K>0$ be a number smaller than $\operatorname{Area}\left(C_{i}\right)$ for each $i$. For any point $[A] \in B( \pm v, K)$, the height of the cylinder $A \cdot C_{i}$ is larger than $|A \cdot( \pm v)|$. For such an $[A]$, any saddle period in $A \cdot\left(X_{0}, q_{0}\right)$ not parallel to $A \cdot( \pm v)$ is longer than $A \cdot( \pm v)$. The saddle periods parallel to $A \cdot( \pm v)$ are also longer than $A \cdot( \pm v)$ since $S$ is supported by an ellipse, so $A \cdot( \pm v)$ is the unique shortest saddle period on $A \cdot\left(X_{0}, q_{0}\right)$. The point $[A]$ is in $H_{m}(S)$.

Proposition 3.18. Suppose $L$ has finite volume. The set $L[1]$ has $\# C(L)$ connected components, each of which is diffeomorphic to the annulus and L[1] contains a horoball around each cusp of $L$.

Proof. By Proposition 3.9, each connected component of $L[1]$ is equal to $f\left(H_{m}(S)\right)$ for some $S=\{ \pm v\} \in \operatorname{Ell}\left(X_{0}, q_{0}\right)[1]$. By Theorem 3.15, $\pm v$ is stabilized by a unipotent element of $\operatorname{PSL}\left(X_{0}, q_{0}\right)$, and $\operatorname{Stab}(S)$ is isomorphic to $\mathbb{Z}$. The component $f\left(H_{m}(S)\right)$ is diffeomorphic to the annulus $H_{m}(S) / \operatorname{Stab}(S)$.

To see that $L[1]$ has $\# C(L)$ connected components, note that the equality of sets $T_{1}=T_{3}$ from Proposition 3.16 gives a bijection between the cusps of $\operatorname{PSL}\left(X_{0}, q_{0}\right)$ and elements of $\operatorname{Ell}\left(X_{0}, q_{0}\right)[1]$ which is equivariant with respect to $\operatorname{PSL}\left(X_{0}, q_{0}\right)$. So we have:

$$
\# C(L)=\# C\left(\operatorname{PSL}\left(X_{0}, q_{0}\right)\right) / \operatorname{PSL}\left(X_{0}, q_{0}\right)=\# \operatorname{Ell}\left(X_{0}, q_{0}\right)[1] / \operatorname{PSL}\left(X_{0}, q_{0}\right)
$$

and the right hand side is in bijection with the number of components of $L[1]$ by Proposition 3.9.

Now fix a cusp $c$ of $L$ and let $t \in \partial \mathbb{H}$ be any cusp of $\operatorname{PSL}\left(X_{0}, q_{0}\right)$ mapping to $c$. By Proposition 3.16, $t=-x / y$ for some $S=\{ \pm(x+i y)\} \in \operatorname{Ell}\left(X_{0}, q_{0}\right)$. The set $H_{m}(S)$, whose image under $f$ lies in $L[1]$, contains a horoball about $t$ by Proposition 3.17.

Proposition 3.19. If the volume of $L$ is finite, then $\operatorname{Spine}_{W G}(L)$ is compact, the set $\bigcup_{n \geq 3} L[n]$ is finite, the set $L[2]$ has finitely many components.

Proof. By the previous proposition, the set $L[1]$ contains a neighborhood of each cusp in $L$. The spine $\operatorname{Spine}_{W G}(L)$ is closed by Proposition 2.3 and is contained in a compact subset of $L$ since it is contained in the complement of $L[1]$. Since $\bigcup_{n \geq 3} L[n]$ is a discrete subset of this compact set, $\bigcup_{n \geq 3} L[n]$ is finite. Since $L$ has finite volume, the Euler characteristic of $L$ is finite. The homotopy equivalent set $\bigcup_{n>2} L[n]$ also has finite Euler characteristic. But the Euler characteristic of $\bigcup_{n \geq 2} L[n]$ is equal the difference between the number of points in $\bigcup_{n \geq 3} L[n]$ and the number of components of $L$ [2] (and possibly a finite contribution from the orbifold points on $L$ ) so $L$ [2] has finitely many components. 
Proof of Theorem 1.3. The remaining claims about $L[n]$ when $L$ has finite volume are established in Propositions 3.18 and 3.19.

Algorithm. Fix a point $[X, q] \in L[k]$ with $k \geq 3$. We conclude this section by describing an algorithm for finding the neighbors of $[X, q]$ in the graph whose vertices are points in $\bigcup_{n \geq 3} L[n]$ and whose edges are components of $L[2]$.

Choose a matrix $A \in G$ so $[X, q]=\left[A \cdot\left(X_{0}, q_{0}\right)\right]$ and set $S=A^{-1} \operatorname{Per}_{m}(A$. $\left.\left(X_{0}, q_{0}\right)\right)$ so $H_{m}(S)=\{[A]\}$ and $f\left(H_{m}(S)\right)=[X, q]$. Let $\pm v_{1}, \ldots, \pm v_{k}$ be the elements in $S$ ordered by the counterclockwise cyclic ordering induced by the unique ellipse supporting $S$. The components of $L[2]$ emanating from $[X, q]$ are the geodesic arcs $f\left(H_{m}\left(\left\{ \pm v_{i}, \pm v_{i+1}\right\}\right)\right)$, where subscripts are read modulo $k$.

One endpoint of $H_{m}\left(\left\{ \pm v_{j}, \pm v_{j+1}\right\}\right)$ is $H_{m}(S)$. To compute the other endpoint of $H_{m}\left(\left\{ \pm v_{j}, \pm v_{j+1}\right\}\right)$ :

(1) Compute the matrix $B \in G$ with $B \cdot\left( \pm v_{j}\right)= \pm 1$ and $B \cdot\left( \pm v_{j+1}\right)= \pm i$. The cyclic ordering on the $v_{i}$ 's ensures that $B \cdot S$ lies in the quadrant $\{ \pm(x+i y)$ : $x y \leq 0\}$.

(2) Find the minimum $\mathrm{Ecc}_{0}$ of the quantity:

$$
\operatorname{Ecc}( \pm w)=\left(1-x^{2}-y^{2}\right) /(2 x y) \text { where } \pm(x+i y)=B \cdot( \pm w)
$$

and $\pm w$ ranges in the saddle periods for which $B \cdot( \pm w)= \pm(x+i y)$ with $|x-y|<1$. The condition on $\pm w$ ensures there is an ellipse $E$ passing through $\pm 1, \pm i$ and $B \cdot( \pm w)$ and the quantity $\operatorname{Ecc}( \pm w)$ measures the eccentricity of $E$.

(3) The collection of saddle periods:

$S^{\prime}=\left\{ \pm w \in \operatorname{Per}\left(X_{0}, q_{0}\right): B \cdot( \pm w)= \pm(x+i y)\right.$ with $\left.x^{2}+2 \operatorname{Ecc}_{0} x y+y^{2}=1\right\}$

contains at least three saddle periods and is supported by an ellipse. The endpoints of $H_{m}\left(\left\{ \pm v_{j}, \pm v_{j+1}\right\}\right)$ are $H_{m}(S)$ and $H_{m}\left(S^{\prime}\right)$.

Figure 2 gives an example of a quadratic differential up to scale with $\# N(X, q)=3$, as well as its three neighbors. 


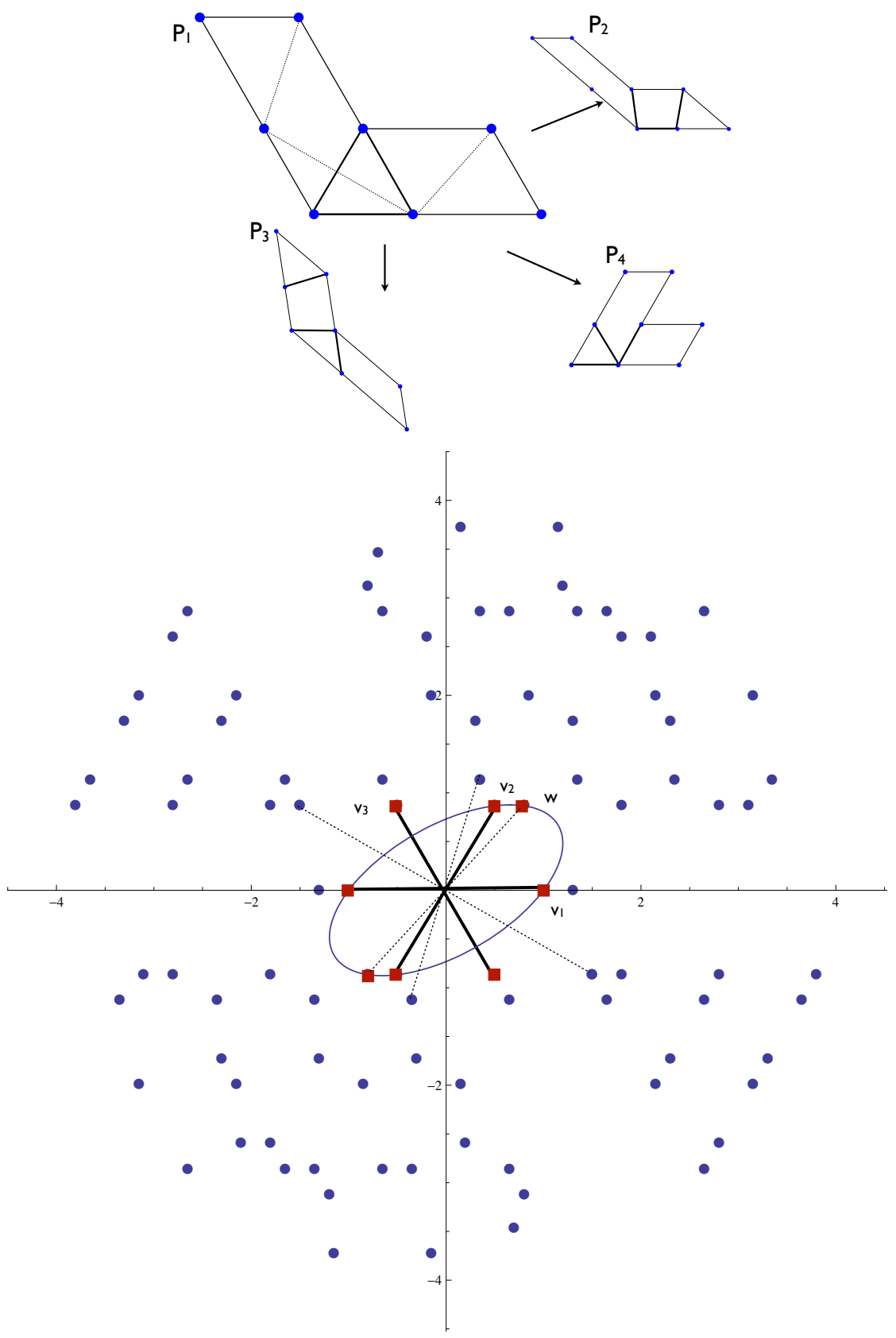

Figure 2. Gluing parallel sides of equal length on the polygons (top) gives four genus two differentials $\left(X_{i}, q_{i}\right)=\left(P_{i}, d z^{2}\right) / \sim$ with $\# N\left(X_{i}, q_{i}\right)=3$. The thick lines in $P_{i}$ give the shortest saddle connections on $\left(X_{i}, q_{i}\right)$. The three neighbors of the differential up to scale $\left[X_{1}, q_{1}\right]$ are $\left[X_{i}, q_{i}\right]$ for $i \geq 2$ as is easily computed by enumerating a small number of the saddle periods on $\left(X_{1}, q_{1}\right)$ (bottom, generated by code written by C. McMullen). 


\section{Examples}

In this section, we study several examples of Veech groups in genus two using our algorithm.

L-shaped polygons. Each integer $D \geq 5$ satisfying $D \equiv 0$ or $1 \bmod 4$ determines a Euclidean octagon $P(D)$ as in Figure 3. Gluing parallel sides of equal length together by translations gives a genus two Riemann surface with a holomorphic quadratic differential:

$$
\left(X_{D}, q_{D}\right)=\left(P(D), d z^{2}\right) / \sim .
$$

An important result of Calta and McMullen is that the Veech group of $\left(X_{D}, q_{D}\right)$ is a lattice:

Theorem 4.1 (Calta, McMullen). The Veech group $\operatorname{PSL}\left(X_{D}, q_{D}\right)$ is a lattice.

Set $Q L_{D}=G \cdot\left(X_{D}, q_{D}\right)$ and $L_{D}=\mathbb{C}^{*} \backslash Q L_{D}$.

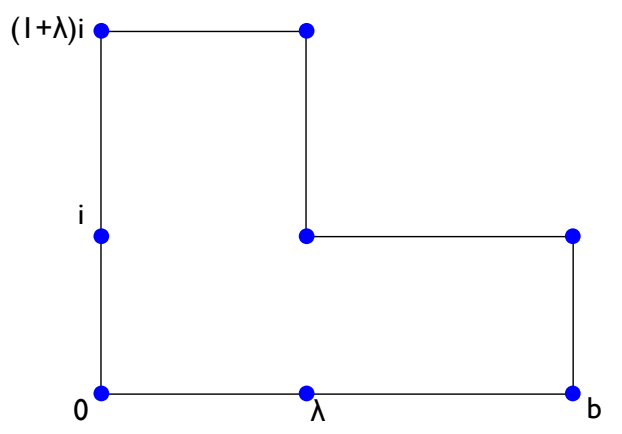

Figure 3. Each integer $D \geq 5$ with $D \equiv 0$ or $1 \bmod 4$ determines a Euclidean octagon $P(D) \subset \mathbb{C}$ built out of a square of side length $\lambda=\frac{e+\sqrt{D}}{2}$ and a $b \times 1$-rectangle where $b=\left(D-e^{2}\right) / 4$ and $e=-1$ or 0 so that $e \equiv D \bmod 2$. Gluing parallel sides of equal length together by translations gives a genus two quadratic differential $\left(X_{D}, q_{D}\right)=\left(P(D), d z^{2}\right) / \sim$ with lattice Veech group.

Fundamental domains and generators. We now describe how to encode an ideal $n$-gon $D(S)$ and generators $\Gamma_{(S, \sigma)}$ for a Fuchsian group with fundamental domain $D(S)$ by a pair $(S, \sigma)$ consisting of a finite set $S \subset \mathbb{R}^{2}$ and a gluing involution $\sigma$ in the permutation group of $S$.

Let $v_{1}=\left(\begin{array}{l}x_{1} \\ y_{1}\end{array}\right), \ldots, v_{n}=\left(\begin{array}{l}x_{n} \\ y_{n}\end{array}\right)$ be the elements of $S$ ordered so that $-x_{1} / y_{1}<$ $-x_{2} / y_{2}<\cdots<-x_{n} / y_{n}$ and set $z_{j}=x_{j}+i y_{j}$. Define $D(S) \subset \mathbb{H}$ to be the convex hull of the set $\left\{-x_{1} / y_{1}, \ldots,-x_{n} / y_{n}\right\} \subset \partial \mathbb{H}$. 
Given a pair $v_{j}$ and $v_{k}$ in $S$, there is a unique element $A\left(v_{j}, v_{k}\right) \in G$ satisfying:

$$
A\left(v_{j}, v_{k}\right) \cdot\left( \pm\left(z_{j}\right)\right)= \pm z_{k+1} \text { and } A\left(v_{j}, v_{k}\right) \cdot\left( \pm z_{j+1}\right)= \pm z_{k}
$$

The Möbius transformation $m_{A\left(v_{i}, v_{j}\right)}$ takes the edge of $D(S)$ connecting $-x_{j} / y_{j}$ to $-x_{j+1} / y_{j+1}$ to the edge connecting $-x_{k+1} / y_{k+1}$ to $-x_{k} / y_{k}$. If $\sigma$ is an order two element in the permutation group of $S$, the set:

$$
\Gamma_{(S, \sigma)}=\left\{A\left(v_{i}, \sigma\left(v_{i}\right)\right): v_{i} \in S\right\}
$$

generates a Fuchsian group with $D(S)$ as fundamental domain.

Example: $\boldsymbol{D}=12$. The Riemann surface $L_{12}$ has genus zero, three cusps and one orbifold point whose orbifold order is two. Using our algorithm, we enumerated the components of $L_{12}[n]$ for $n \geq 2$. The set $L_{12}$ [3] consists of three differentials up to scale, $L_{12}[n]$ is empty for $n \geq 4$ and $L_{12}[2]$ has five components.

We also computed a pair $\left(S_{12}, \sigma_{12}\right)$ encoding a fundamental domain and generators for $\operatorname{PSL}\left(X_{12}, q_{12}\right)$ :

$$
S_{12}=\left\{\left(\begin{array}{c}
\sqrt{3} \\
1
\end{array}\right),\left(\begin{array}{c}
3-\sqrt{3} \\
1
\end{array}\right),\left(\begin{array}{l}
0 \\
1
\end{array}\right),\left(\begin{array}{c}
\sqrt{3}-3 \\
1
\end{array}\right),\left(\begin{array}{c}
3-\sqrt{3} \\
0
\end{array}\right)\right\} \text { and } \sigma_{12}=(1,1)(2,3)(4,5) .
$$

The domain $D\left(S_{12}\right)$ is the convex hull of $\{-\sqrt{3},-3+\sqrt{3}, 0,3-\sqrt{3}, \infty\}$ and $\operatorname{PSL}\left(X_{12}, q_{12}\right)$ is generated by:

$$
\Gamma_{\left(S_{12}, \sigma_{12}\right)}=\left\{ \pm\left(\begin{array}{cc}
3+2 \sqrt{3}-3-4 \sqrt{3} \\
2+\frac{4}{\sqrt{3}}-3-2 \sqrt{3}
\end{array}\right), \pm\left(\begin{array}{cc}
1 & 0 \\
-1-\frac{1}{\sqrt{3}} & 1
\end{array}\right), \pm\left(\begin{array}{cc}
1 & -3 \\
0 & 1
\end{array}\right)\right\} .
$$

The domain $D\left(S_{12}\right)$ is depicted in Figure 4.

Example: $\boldsymbol{D}=13$. The Riemann surface $L_{13}$ has genus zero, three cusps, one orbifold point whose orbifold order is two. Using our algorithm, we enumerated the components of $L_{13}[n]$ for $n \geq 2$. The set $L_{13}$ [3] (see Figure 4) consists of three differentials up to scale, $L_{13}[n]$ is empty for $n \geq 4$ and $L_{13}$ [2] has five components.

We also computed a pair $\left(S_{13}, \sigma_{13}\right)$ encoding a fundamental domain and generators for $\operatorname{PSL}\left(X_{13}, q_{13}\right)$ :

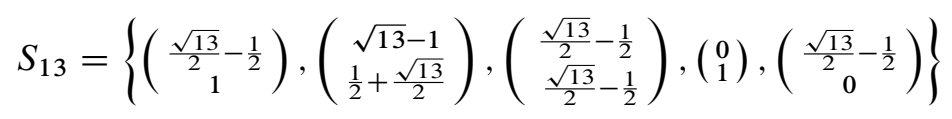

and

$$
\sigma_{13}=(1,2)(3,5)(4,4) .
$$


The domain $D\left(S_{13}\right)$ is the convex hull of $\left\{\frac{1-\sqrt{13}}{2}, \frac{-7+\sqrt{13}}{3},-1,0, \infty\right\}$ and $\operatorname{PSL}\left(X_{13}, q_{13}\right)$ is generated by:

$$
\Gamma_{\left(S_{13}, \sigma_{13}\right)}=\left\{\begin{array}{c} 
\pm\left(\begin{array}{cc}
0 & \frac{1}{2}(1-\sqrt{13}) \\
\frac{1}{6}(1+\sqrt{13}) & 0
\end{array}\right), \pm\left(\begin{array}{cc}
0 & \frac{1}{2}(1-\sqrt{13}) \\
\frac{1}{6}(1+\sqrt{13}) & \frac{1}{2}(-1-\sqrt{13})
\end{array}\right), \\
\pm\left(\begin{array}{cc}
7+2 \sqrt{13} & -2(5+\sqrt{13}) \\
\frac{1}{6}(41+11 \sqrt{13}) & -9-2 \sqrt{13}
\end{array}\right)
\end{array}\right\} .
$$

The domain $D\left(S_{13}\right)$ and the stratification $L_{13}=\bigcup_{n \geq 1} L_{13}[n]$ are drawn in Figure 4 .

$L_{12}$
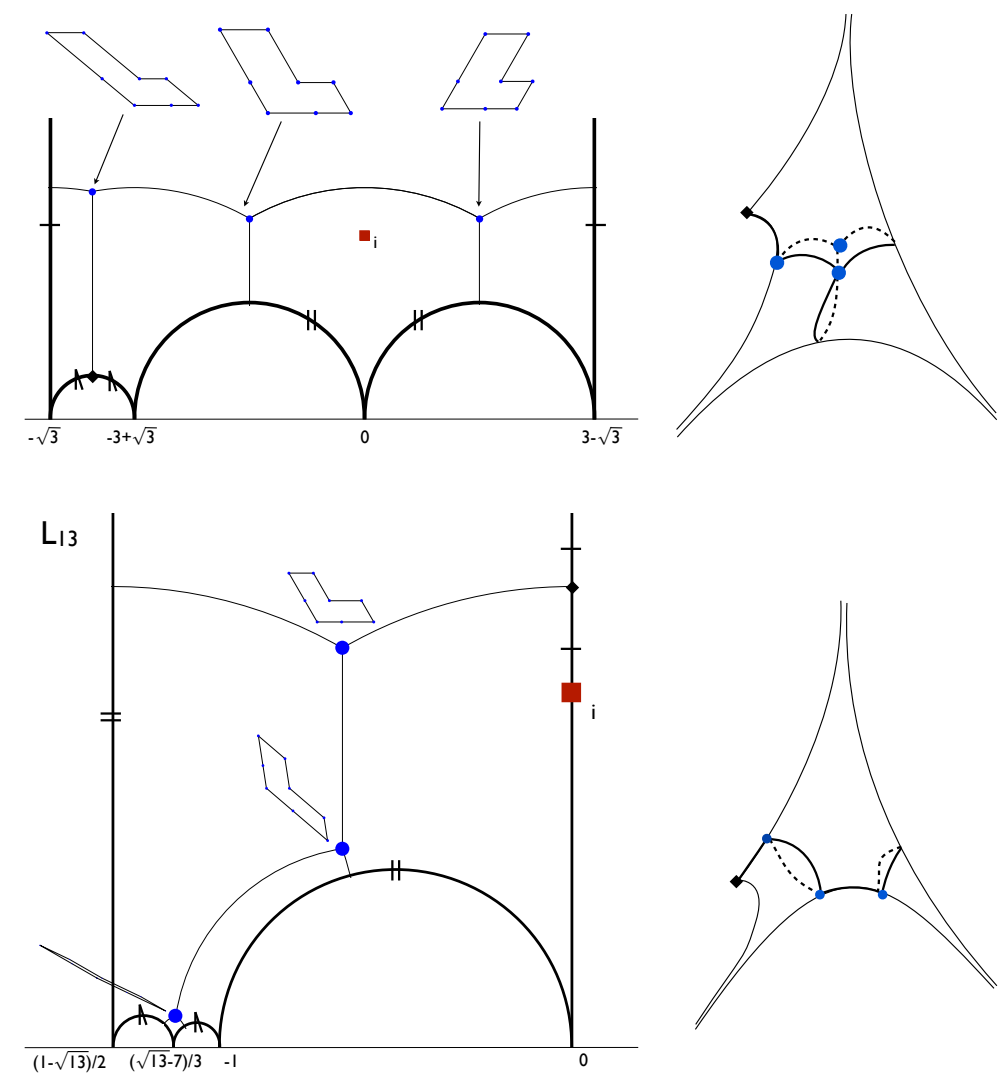

Figure 4. The Riemann surfaces $L_{D}$ for $D=12$ (top) and $D=13$ (bottom) both have genus zero, three cusps and one orbifold point of order two. The stratification $L_{D}=\bigcup_{n \geq 1} L_{D}[n]$ decomposes $L_{D}$ into easy to understand pieces. Enumerating points in $L_{D}[n]$ for $n \geq 3$ and components of $L_{D}$ [2] allows us to compute a fundamental domain and generators for $\operatorname{PSL}\left(X_{D}, q_{D}\right)$. 
Example: $\boldsymbol{D}=44$. The Riemann surface $L_{44}$ has Euler characteristic $-21 / 2$, genus one, nine cusps and three orbifold points each of which has orbifold order two. The set $L_{44}[n]$ is empty for $n \geq 4$, the set $L_{44}$ [3] consists of 21 points, and the set $L[2]$ has 33 connected components.

The pair $\left(S_{44}, \sigma_{44}\right)$ listed in Table 1 encodes a fundamental domain and generators for $\operatorname{PSL}\left(X_{44}, q_{44}\right)$. The domain $D\left(S_{44}\right)$ is the ideal 23-gon equal to the convex hull of:

$\left\{\begin{array}{c}-11+\sqrt{11},-\frac{11}{2},-\sqrt{11}, \frac{1}{3}(-11+\sqrt{11}), \frac{1}{7}(-11-2 \sqrt{11}), \frac{4}{7}(-11+2 \sqrt{11}), \frac{1}{5}(-22+3 \sqrt{11}), \\ \frac{3}{10}(-11+\sqrt{11}),-2, \frac{1}{5}(-33+7 \sqrt{11}), \frac{1}{4}(-11+\sqrt{11}), \frac{1}{7}(-22+3 \sqrt{11}), \frac{1}{5}(-11+\sqrt{11}), 0, \frac{1}{5}(11-\sqrt{11}), \\ \frac{1}{4}(11-\sqrt{11}), \frac{1}{5}(33-7 \sqrt{11}), 2,-\frac{3}{10}(-11+\sqrt{11}), \frac{1}{5}(22-3 \sqrt{11}), \frac{1}{3}(11-\sqrt{11}), \sqrt{11}, \infty\end{array}\right\}$

The domain $D\left(S_{44}\right)$ and the preimages of $L_{44}[n]$ for $n \geq 2$ are drawn in Figure 1. A few examples of elements the Veech group of $\left(X_{44}, q_{44}\right)$ include:

$\pm\left(\begin{array}{cc}1 & -11 \\ 0 & 1\end{array}\right), \pm\left(\begin{array}{cc}5+2 \sqrt{11} & -11-6 \sqrt{11} \\ 2+\frac{8}{\sqrt{11}} & -5-2 \sqrt{11}\end{array}\right), \pm\left(\begin{array}{cc}25+6 \sqrt{11} & -6(11+2 \sqrt{11}) \\ -10-\frac{30}{\sqrt{11}} & 25+6 \sqrt{11}\end{array}\right) \in \operatorname{PSL}\left(X_{44}, q_{44}\right)$.

A full list of generators for $\operatorname{PSL}\left(X_{44}, q_{44}\right)$ can be easily computed from the pair $\left(S_{44}, \sigma_{44}\right)$ listed in Table 1. 


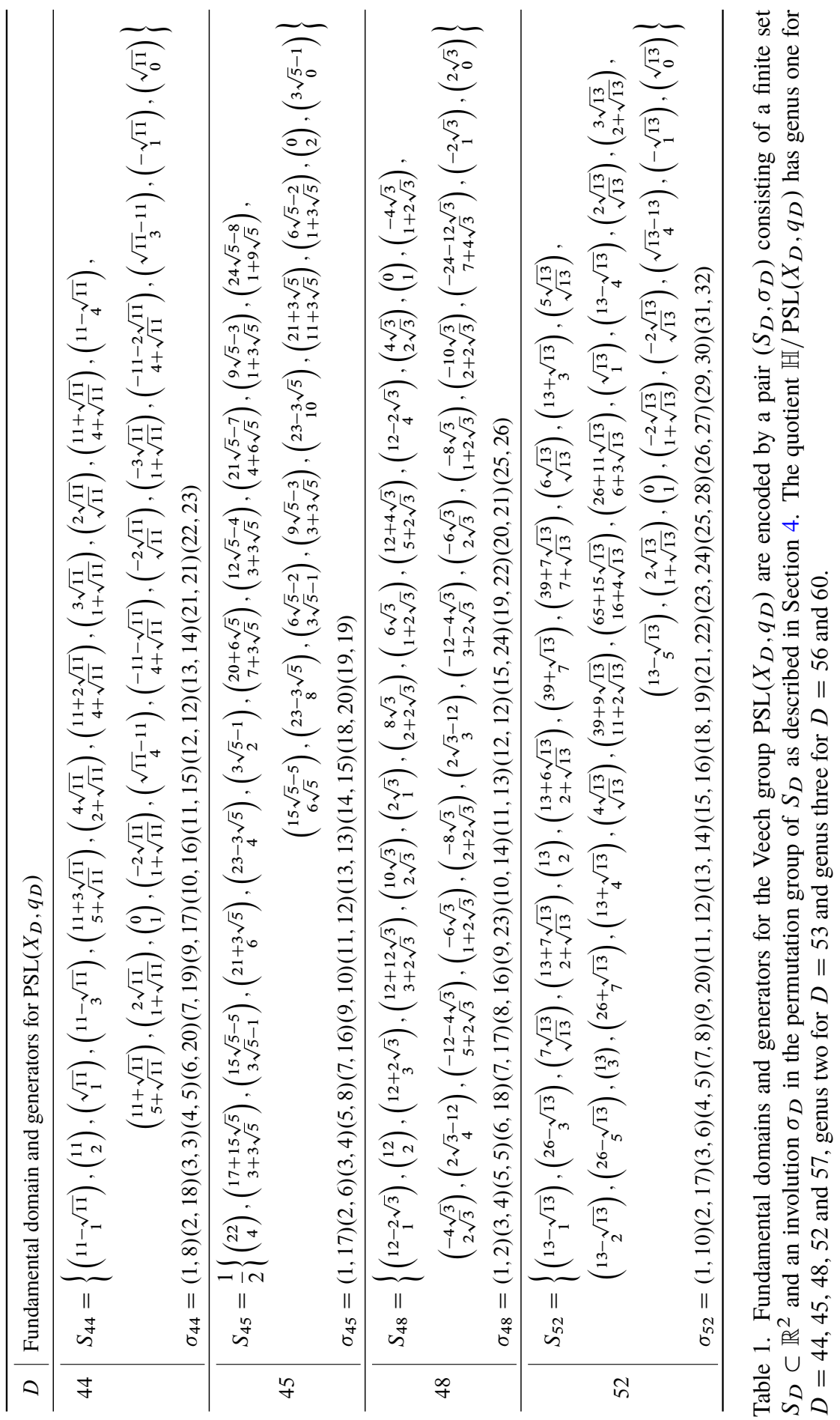




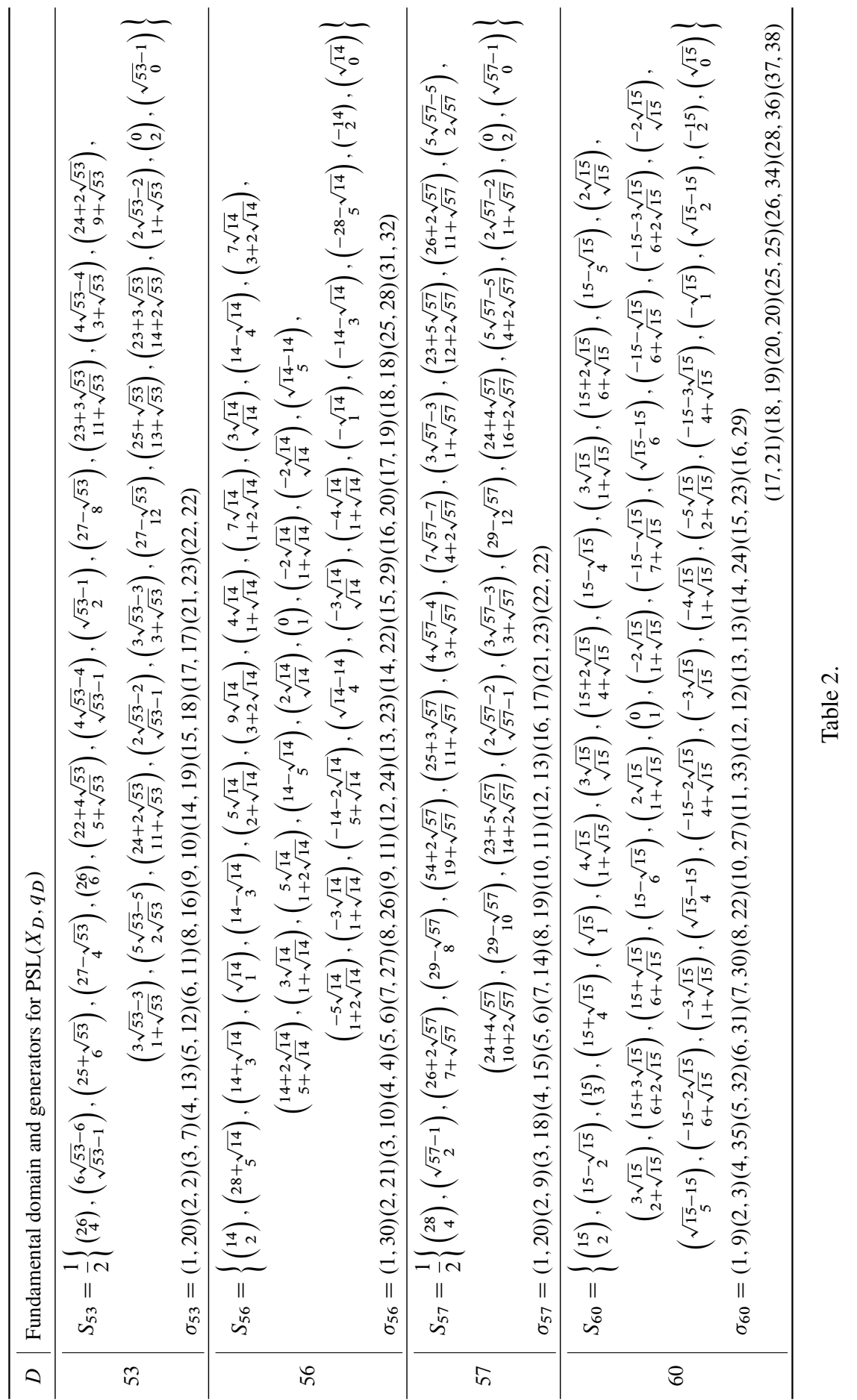




\section{References}

[1] A. Ash, Small-dimensional classifying spaces for arithmetic subgroups of general linear groups, Duke Mathematical Journal, 51 (1984), 459-468. Zbl 0542.22011 MR 0747876

[2] M. Bainbridge, Euler characteristics of Teichmüller curves in genus two, Geom. Topol., 11 (2007), 1887-2073. Zbl 1131.32007 MR 2350471

[3] B. Bowditch and D. Epstein, Natural triangulations associated to a surface, Topology, 27 (1988), 91-117. Zbl 0649.32017 MR 0935529

[4] J. P. Bowman, Teichmüller geodesics, Delaunay triangulations, and Veech groups, preprint.

[5] K. Calta, Veech surfaces and complete periodicity in genus two, J. Amer. Math. Soc., 17 (2004), 871-908. Zbl 1073.37032 MR 2083470

[6] J. S. Ellenberg and D. B. McReynolds, Arithmetic Veech sublattices of SL(2, Z), Duke Math. J., 161 (2012), 415-429. Zbl 1244.32009 MR 2881227

[7] P. Hubert and E. Lanneau, Veech groups without parabolic elements, Duke Mathematical Journal, 133 (2006), 335-346. Zbl 1101.30044 MR 2225696

[8] P. Hubert and T. A. Schmidt, Infinitely generated Veech groups, Duke Mathematical Journal, 123 (2004), 49-70. Zbl 1056.30044 MR 2060022

[9] H. Masur and S. Tabachnikov, Rational billiards and flat structures, in Handbook of dynamical systems, Vol. 1A, 1015-1089, North-Holland, Amsterdam, 2002. Zbl 1057.37034 MR 1928530

[10] C. T. McMullen, Billiards and Teichmüller curves on Hilbert modular surfaces, J. Amer. Math. Soc., 16 (2003), 857-885. Zbl 1030.32012 MR 1992827

[11] C. T. McMullen, Teichmüller geodesics of infinite complexity, Acta Math., 191 (2003), 191-223. Zbl 1131.37052 MR 2051398

[12] C. T. McMullen, Teichmüller curves in genus two: Discriminant and spin, Math. Ann., 333 (2005), 87-130. Zbl 1086.14024 MR 2169830

[13] R. E. Mukamel, Orbifold points on Teichmüller curves and Jacobians with complex multiplication, preprint.

[14] R. E. Mukamel, Triangulated surfaces in Sage [computer file], retrieved 2014. http: //math.uchicago.edu/ ronen/software/triangulated_surface.tar.gz

[15] G. Schmithüsen, An algorithm for finding the Veech group of an origami, Experimental Mathematics, 13 (2004), 459-472. Zbl 1078.14036 MR 2118271

[16] J. Smillie and B. Weiss, Characterizations of lattice surfaces, Invent. Math., 180 (2010), 535-557. Zbl 1195.57041 MR 2609249

[17] W. P. Thurston, On the geometry and dynamics of diffeomorphisms of surfaces, Bull. Amer. Math. Soc., 19 (1988), 417-431. Zbl 0674.57008 MR 0956596

[18] W. A. Veech, Teichmüller curves in moduli space, Eisenstein series and an application to triangular billiards, Invent. Math., 97 (1989), 553-583. Zbl 0676.32006 MR 1005006

[19] W. A. Veech, Bicuspid F-structures and Hecke groups, Proc. London Math. Soc., 103 (2011), 710-745. Zbl 1238.32013 MR 2837020 
Vol. 92 (2017) Fundamental domains and generators for lattice Veech groups

[20] A. Zorich, Flat surfaces, in Frontiers in Number Theory, Physics and Geometry. Volume 1: On random matrices, zeta functions and dynamical systems, 439-586, Springer-Verlag, Berlin, 2006. Zbl 1129.32012 MR 2261104

Received December 02, 2014

R. Mukamel, Department of Mathematics, Rice University, 6100 Main St., Houston, TX 77005, USA

E-mail: ronen@rice.edu 